\title{
Mathwise Astronomy and the TLTP: aiding or degrading education?
}

\author{
By E.M. McCabe
}

School of Mathematical Studies, University of Portsmouth, UK

\section{Background}

The Teaching and Learning Technology Programme (TLTP) in the UK was launched in 1992 to "develop innovations in teaching and learning through the power of technology". Increasing numbers of students with mixed abilities and backgrounds were entering into higher education. Flexible course structures and the need for remedial teaching added further motivation in the search for methods of improving productivity and efficiency.

Since 1992 over 33 million of funding has been awarded to 76 projects spanning the university curriculum. When support from host institutions is taken into account, overall funding for the TLTP is estimated at 75 million. TLTP materials are now becoming available to assist institutions in maintaining and enhancing the quality of their teaching provision. The successful implementation of this new technology is requiring each institution to rethink its teaching and learning strategies (Laurillard, 1993).

Approximately one quarter of the projects are based on a single institution and are concerned with the culture change, the integration of technology and staff development. The remainder are consortia concerned with courseware development and involve staff from between two and fifty universities.

Astronomy is represented within one of the largest consortia, the UK Mathematics Courseware Consortium (UKMCC), which has received 1.3 million of TLTP funding. Other projects include Software Teaching of Modular Physics (SToMP) and Statistics Education through Problem Solving (STEPS).

\section{UK Mathematics Courseware Consortium}

Mathwise, the product of the UKMCC, is an exciting new computer-based learning environment for students of mathematics in the sciences and engineering (Beilby, 1993 and Harding, 1996). A set of fifty modules in foundation mathematics and its applications are being developed. The first thirty, including the astronomy module, were completed by December 1995 and the remaining twenty are scheduled for completion by December 1996.

\section{Phase 1}

Foundation

Applications
20 modules, e.g. Standard Conics

10 modules, e.g. Astronomy

\section{Phase 2}

10 modules, e.g. Vector Calculus $(\times 2)$

10 modules, e.g. Sports Maths

First year undergraduates in science and engineering are the target audience, although topics at higher and lower levels are covered. The foundation modules provide full coverage of the SEFI $\dagger$ core curriculum in mathematics for the European engineer (Barry, 1992). Astronomy was identified as an exceptional application for motivating science and engineering students to explore and understand its underlying mathematics. As a result, the astronomy material considerably exceeds the nominal five hours of study time allocated to each module.

The project has five main support sites for the project development, namely the universities at Birmingham (lead site), Cambridge (Hypercard), Coventry (Toolbook), Heriot-Watt (Authorware and assessment) and Keele (multimedia). While Mathwise Astronomy has been developed for the PC using Toolbook, other modules have been developed using Authorware

$\uparrow$ Society for the Education of the European Engineer 
for the PC/Mac or Hypercard for the Mac. Conversions between Toolbook and Hypercard have been completed for some modules. In all, around forty authors at thirty different universities have developed the courseware. The academics have been responsible for writing, designing and authoring the software to a common specification using templates and tools developed by the support sites.

Universities within the UK can use all Mathwise modules freely and at minimal cost. The first evaluation CD-ROM was released in March 1995. An extended version is currently being distributed by the Numerical Algorithms Group (NAG Ltd.) of Oxford. Several international publishers have expressed their interest and it is expected that the material will become more widely available on CD-ROM in 1997.

\section{Mathwise Astronomy}

Mathwise Astronomy (McCabe, 1994, 1995a) is a spectacular demonstration of the powerful features of Mathwise. Many other astronomical CD-ROM products, such as Redshift 2, are the software equivalent of coffee-table books. They are extremely attractive and interactive, but do not offer a deeper understanding (Howarth, 1996). Several astronomical textbooks offer practical exercises using photograph measurements (Brück, 1990), supplied datasets or suggested observations, but are less interactive. Mathwise Astronomy provides outstanding opportunities for exploring and understanding the Universe, by combining the better features of the interactive CD-ROM and the practical textbook.

The astronomy module is divided into six learning units (Grand Tour of the Universe, Distances to Planets and Stars, Mass of the Sun and Stars, Luminosity and Temperature of Stars, Star Formation and Stellar Evolution) and thirteen leaflets (e.g. Population Density of Stars, Reflecting Telescope Mirror, Escape Velocity, Small Angle Approximations, Energy of a Gas Molecule, Stellar Magnitudes, Derive Kepler's 1st / 2nd / 3rd Law, Powers of Ten).

The learning units have a predominantly linear structure, likened to lectures. Leaflets 'bud' from and return to the main learning units, providing opportunities for further investigation and explanation. Leaflets can also be explored from within the learning units of other foundation or application modules and are likened to drop-in tutorials.

$\begin{array}{ll}\text { module } & \text { lecture course } \\ \text { learning unit } & \text { lecture } \\ \text { leaflet } & \text { tutorial } \\ \text { resource } & \text { library books and programs }\end{array}$

The resources available include References (glossary, maths handbook, biographies and bibliographies) and Tools (calculator, grapher, star maps, simulations, and experiments).

Screen-based observations of the motions of the planets and stars lead on to an understanding of how distances, masses, temperatures and luminosities can be determined. Accurate simulations of astronomical phenomena, such as solar and lunar eclipses, the phases of Venus and stellar parallax, are frequently exploited to provide personalised data. Collapsing interstellar gas clouds and the changing appearance of stars in the H-R diagram allow a basic mathematical understanding of star formation and stellar evolution to be developed.

There are over two hundred screens of highly interactive multimedia. Video clips recorded by Dr. Patrick Moore introduce each learning unit and animations illustrating the astronomy or mathematics appear on most screens (Figure 1).

\section{Computer Aided Virtual Experiments (CAVEs)}

The Mathwise astronomer is encouraged to make observations, take and record measurements, analyse the measurements, derive empirical results, put forward hypotheses, develop mathematical theories and derive analytical results. This idealised scientific method guides the discovery and helps to guarantee success on a reasonable time-scale. The use of a simulation in 

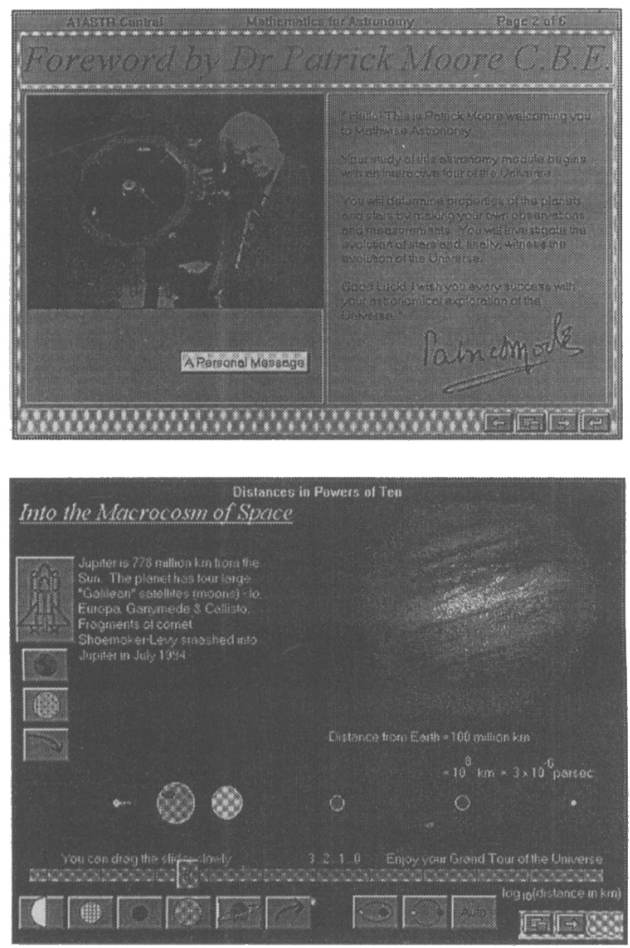

FIGURE 1. Introducing the Grand Tour of the Universe

this context is described as a Computer Aided Virtual Experiment or CAVE. Resources include a wide range of CAVEs for projectiles and celestial orbits (Figure 2).

The dimensions and separations of objects on the screen are measured by 'rubber- banding'. The measurement is recorded continuously while the mouse is dragged and is fixed when the mouse is released. For example, the technique has been used to allow screen measurement of angular separations and diameters, distances of planets from the Sun and parallaxes. Errors occur naturally when making the on-screen measurements, although their origin is quite different from 'real measurement'errors. Data gathered in this way can be used subsequently for the application of mathematical and statistical techniques, which deal with the experimental error.

Time intervals during experiments can be measured by a digital stopwatch with start/stop and reset buttons. The speed of some simulations are controlled by the user, while sensible speeds are fixed for others. An option to allow the use of standard datasets as an alternative to making measurements is generally provided.

Mathwise astronomers can investigate the parameters which govern telescope mirrors, celestial trajectories, stellar properties, star formation or stellar evolution, establish mathematical relationships from their screen measurements and derive theoretical results.

\section{Flexible Study Modes}

The stages in any calculation encourage thought and offer appropriate feedback, in such a way that even a mathematical proof becomes visually exciting! Interactive calculations and examples are illustrated numerically, algebraically, geometrically and graphically in both two and three dimensions.

Within many of the mathematical pages, flexible study modes are permitted (McCabe, 1995b). A reader can choose between a Study mode (cf. a manually driven car), a Revision mode (cf. an automatic car) or a Skim mode (cf. a car in overdrive). The screen is displayed either 

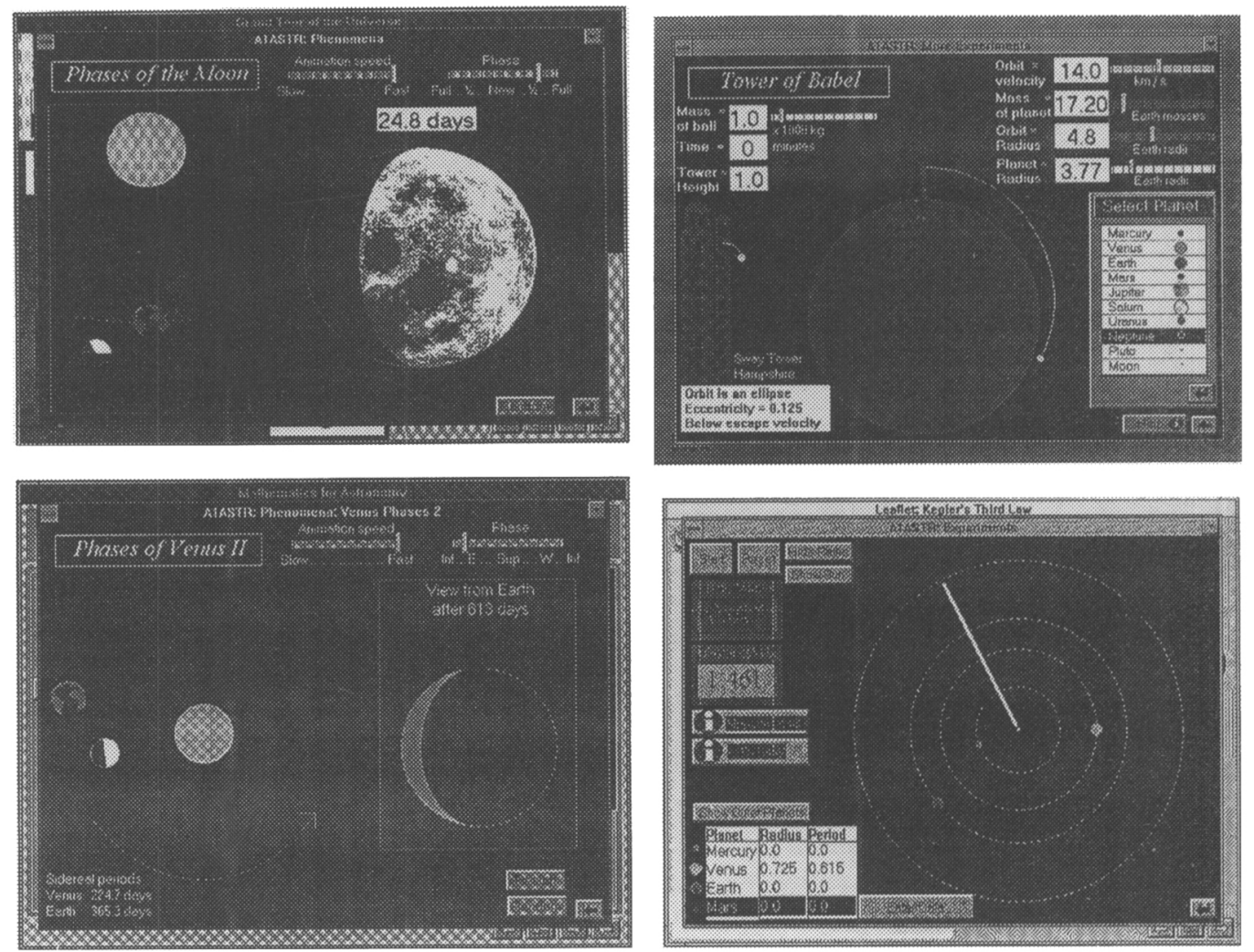

FIgURE 2. A selection of Computer Aided Virtual Experiments (CAVEs)

step-by-step under user control, automatically at a fixed speed or with full details displayed immediately. These options are especially useful for presenting algebraic derivations or proofs, where a careful explanation is required at first reading, but only the final results need to be accessed at a later stage.

Figure 3 shows how a mathematical result is broken up into stages by using a set of menu items. By clicking on each step, the next stage in the reasoning is shown and different parts are highlighted or animated. Any step can be reviewed while following the proof. This technique even allows the reader to investigate the "Grand Evolution of the Universe" from the Big Bang up to the present day!

\section{Evaluation: First Aid or Lemonade ?}

Foundation Mathematics modules are intended to provide remedial materials, encourage revision, extend basic concepts originally taught in the classroom and raise the confidence of the learner. Applications modules are intended to motivate students to acquire the skills and understanding and to provide worked examples which extend the student's technical knowledge to applied problems. A student can receive 'first aid' outside timetabled teaching hours without the fear of failure or embarassment. Under supervision the teacher becomes an ally rather than an opponent. Trials have demonstrated the effectiveness of the techniques used, but with some reservations. 

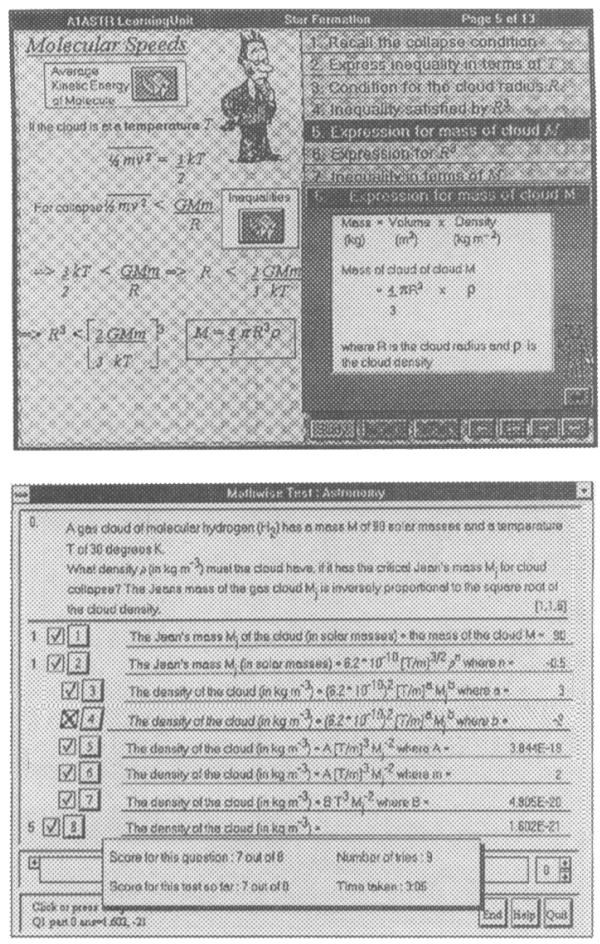

Figure 3. Flexible Study Modes and Structured Response Assessment

There is a natural tendency for students to focus attention on visually attractive simulations and the parts with "fizz". Considerable effort has been made to provide well specified activities with clear objectives. The structure of Mathwise encourages a logical progression, while allowing the option to skip leaflets.

Informal self-assessment is provided within units. A test mechanism for more formal summative assessment has been developed at Heriot-Watt. In common with other Mathwise modules, computer-based questions can be randomly selected from a bank of multiple- choice or structured response questions, which may include several random parameters. Tests may be completed in either a practice or exam mode. In a structured response question a sequence of key parts must be answered, with the option to reveal intermediate steps (Figure 4).

\section{Further Developments}

When used prudently, Mathwise Astronomy and the TLTP are aiding rather than degrading education. They are not an all-embracing panacea, which can eliminate the need for teachers, but they do provide an exciting new dimension to learning, which can significantly improve the quality of education.

Although Mathwise astronomy is designed to be self contained, it is planned to write a series of worksheets to accompany the module. These will provide additional paper- based exercises, which will suggest alternative activities. In some instances it may make the recording of complicated results from screen based simulations more straightforward.

Another TLTP consortium, Software Teaching of Modular Physics SToMP, is also planning to add an astronomy component to its existing open learning materials. The project, involving nine UK universities, uses a flexible multimedia database system, Microcosm, to present and cross-reference information. Adaptation for the World Wide Web and linking to robotic telescopes are further possibilities, but is not yet straightforward for such highly interactive material. 

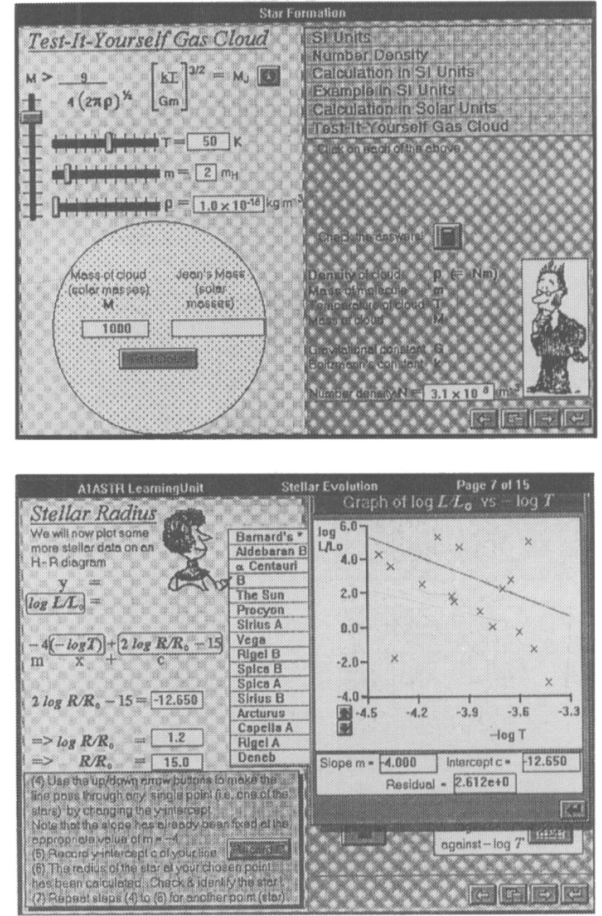

Figure 4. Interactive Stellar Evolution

\section{Acknowledgements}

The support of authors and staff working on the UKMCC Project is gratefully acknowledged.

\section{Further Information}

TLTP products, including Mathwise and SToMP, can be used freely in UK universities.

UKMCC Project, Centre for Computer-Based Learning, University of Birmingham, Edgbaston, Birmingham, UK. B15 2TT

Tel: 01214144800 Fax: 01214146267 email:mathwise@bham.ac.uk

URL: http://www.bham.ac.uk/mathwise/homepage.htm

Software Teaching of Modular Physics, Department of Physics, University of Surrey, Guildford, Surrey, UK.

Tel: 01483259414 Fax: 01483259501 email: stomp@surrey.ac.uk TLTP email: tltp@hefce.ac.uk URL: http://www.icbl.hw.ac.uk/tltp Author email: mccabe@sms.port.ac.uk

\section{REFERENCES}

BARRY, M.J.D., 1992, A Core Curriculum in Mathematics for the European Engineer, SEFI (Society for Education of the European Engineer) document 92.1 .

BeIlBy M.H., 1993, Mathwise, CBL Environment for Mathematics, CTI Maths and Stats Newsletter, Vol. 4 , No. 4 .

BRück, M., 1990, Exercises in Practical Astronomy Using Photographs, Adam Hilger.

Harding, R.D., 1996, Cooperative Cross-Platform Courseware Development, ALT-J, Association for Learning Technology Journal, vol. 4, no. 1, 22 - 27. 
Howarth, I., 1996, Review of Redshift 2, Observatory, vol, 116, no. 1132, 194 - 6.

LaUrillard, D., 1993, Rethinking University Teaching: A Framework for the Effective Use of Educational Technology, Routledge.

MCCABE, E.M., 1994, I in the Sky: Interaction with the Universe in the Mathwise Astronomy Module, UK Toolbook User Conference 94 Proceedings ISBN 086292427 8, 37 - 49.

MCCABE, E.M., 1995a, Sliding into Mathematics: The Creative Use of Widgets for Engineering the Universe, Eurographics UK '95 Conference, Loughborough University, ISBN 09521097 $27233-247$.

MCCABE, E.M., 1995b, The Incorporation of Flexible Learning Modes into Computer Based learning of Mathematics and Astronomy, Hypermedia in Sheffield '95, Sheffield Academic Press, 525 - 532, ISBN 1850755728. 